Dhaka Univ. J. Biol. Sci. 24(2): 199-207, 2015 (July)

\title{
HEALTH RELATED QUALITY OF LIFE AND SELF ESTEEM OF SUBSTANCE USERS IN BANGLADESH
}

\author{
Md. Azharul Islam* And Mst. Adiba Akter \\ Department of Educational and Counselling Psychology, \\ University of Dhaka, Dhaka-1000, Bangladesh
}

Key words: Health related quality, Substance use, Self esteem

\begin{abstract}
This study explored health related quality of life (HRQoL) of substance users (SUs) in Bangladesh in comparison with healthy control groups (CGs). Additionally, role of self esteem and sex on HRQoL was investigated. A trained assistant counselling psychologist measured HRQoL and self-esteem of 120 SUs and 120 CGs using SF-36 questionnaire and Rosenberg Self Esteem scale, respectively. SUs were mostly male $(79.2 \%)$ from middle socioeconomic class (94\%). SUs scored poorer in all domains of SF-36 than the CGs. The magnitude of the effect size was largest for the role physical (effect size $=-0.79$ ), general mental health (effect size $=-0.76$ ) and social functioning (effect size $=-0.73$ ) subscale. Overall mental health was lower than physical health (effect size $=-0.897$ ). Males in both groups were better in four sub-scales while females reported less role limitation due to physical and emotional problems. Self esteem moderated HRQoL of both group with slightly higher magnitude for SUs. HRQoL of SU of Bangladesh was lower than the study population.
\end{abstract}

\section{Introduction}

Illicit drug use has been reported as 'significant contributor to the global disease burden (GBD), larger than many mental disorders and greater than all maternal conditions combined'(1). The GBD study 2010 revealed that illicit drug dependence directly accounted for 20.0 million disability-adjusted life years (DALYs) in 2010(2). Globally men are more prone to illicit drugs than women (2). In Bangladesh, substance use (SU) related information is insufficient. Department of Narcotic Control of Bangladesh estimated around 1.5 million people are engaged in illicit drug use while other reported the figure to be 2.5 to 5 million(3).

Health related quality of life (HRQoL) is a pivotal outcome indicator in substance intervention studies and clinical trials ${ }^{(4-7)}$. It is generally defined as 'a subjective perception of the patient's level of physical, emotional and social functioning and wellbeing, as well as its repercussion on his/her daily life'(8). Although HRQoL has been widely examined for various chronic health conditions, studies with SU were relatively

*Author for correspondence: < azharulislam@du.ac.bd>. 
low. Recently evidence is accumulating that HRQoL is related with successful intervention in patients with Opioid dependence ${ }^{(7)}$. Results from six clinical trials on 'maintenance pharmacotherapy' showed clinical and statistically significant progress of participants' HRQoL after three months of intervention(5).

The short form of health 36 (SF-36) ${ }^{(9)}$ is a generic instrument of HRQoL that taps eight dimensions of well-being. O'Brien et al.(5) found that all SF-36 sub-domains of Heroindependents were significantly worse than the Australian norm. In another study Opiate users were found significantly lower than HRQoL for all sub-scales with largest difference observed in role-emotional followed by role-physical and general health ${ }^{(10)}$. Although SU causes damage in every area of HRQoL, the magnitude of difference varied for types of substance and socio-cultural context. Socio-cultural context might affect perception of HRQoL of different SUs. Thus, designing appropriate intervention to foster HRQoL of this group requires culture specific evidence that would tap specific vulnerable area. On the other hand, it has been established that self esteem foster psychological well-being ${ }^{(11)}$. Studies also suggesting self esteem can buffer the harmful effect of SU on HRQoL ${ }^{(11,12)}$. High self esteem was associated with quicker abstinence from SU for adolescent, lower anxiety and longer off-drug period(13).

Although evidences are growing in the western context, similar study is almost absent in Bangladesh. A few descriptive studies reported prevalence and sociodemographic nature of SU among University students ${ }^{(14)}$ and recovering patients ${ }^{15,16)}$ but none of them considered HRQoL of SUs. Such effort in Bangladesh will facilitate intervention by indicating changes in physical, mental and social well-being of various treatment programs. Knowledge on role of self esteem in this relation will also open possible avenue for psychological intervention. This study compared HRQoL of recovering SUs to see their status with normal control group. Additionally, role of self esteem and sex on HRQoL was explored.

\section{Materials and Methods}

Total 120 recovering SU were selected from two drug rehabilitation centres. For comparison, 120 healthy control participants were selected from the nearby areas. A trained assistant counselling psychologist measured HRQoL and self-esteem. Prior to data collection written consent was taken from each participant and concerned authorities.

Bangla version of SF-36 was used to assess HRQoL. This questionnaire has been validated for both clinical samples(17) and general population ${ }^{(18)}$. Eight sub-scales were computed, physical functioning (PF), role limitations due to physical health problems $(\mathrm{RP})$, bodily pain $(\mathrm{BP})$, social functioning $(\mathrm{SF})$, general mental health $(\mathrm{GMH})$, role limitations due to emotional problems (RE), vitality, energy and fatigue (VEF) and general health perceptions (GHP). The scoring of the items varied from dichotomous 
scales (yes/no) to six point ordinal scales. The total score is calculated from the mean of the eight sub-scales ranging from 0 to 100 where a high score indicates better health. Besides, mean of four mental health sub-scales (RE, VEF, GMH and SF) and four physical health sub-scales (RP, BP, PF and GHP) score was calculated to capture mental component summary score (MCS) and physical component summary score (PCS), respectively. The questionnaire has been tested and has satisfactory reliability and validity ${ }^{(17,19)}$.

Bangla adapted ${ }^{(20)}$ Rosenberg Self-Esteem (RSE) scale ${ }^{(21)}$ was used to measure selfesteem. The RSE scale consists of ten items. The answers are scored on a four point Likert scale, giving a range from 10 to 40 . The RSE test-retest correlations are typically in the range of 0.82 to 0.88 , and Cronbach's alpha for various samples are in the range of 0.77 to $0.88^{(21,22)}$. Participant's age, sex, socio-economic status (SES), education, number of siblings, family type and birth order were also recorded.

\section{Results and Discussion}

SUs were slightly older $(M=27.82, S D=7.46)$ than that of CGs $(M=24.93, S D=3.32)$. SU participants were predominantly male $(79.2 \%)$ from middle socioeconomic class (94\%). Two third of the SUs were from nuclear family (66.7\%). One third of SUs had some undergraduate degree while similar number of them reached up to some school education. The birth order of the SUs was almost equally distributed to eldest, middle and youngest categories. The CG on the other side was almost sex balanced, mostly resided in nuclear families $(65.8 \%)$. Nearly half of the CGs had postgraduate education. Over 60 per cent of CGs reported to be from lower SES (Table 1). This finding is consistent with previous studies ${ }^{(16)}$.

All SF-36 sub-scales were positively associated with themselves $(\mathrm{p}<0.01)$. Self esteem was also positively associated with sub-scales but more strongly with GHP $(r=0.53, \mathrm{p}<$ $0.01)$ and GMH $(r=0.405, \mathrm{p}<0.01)$. This refers participants who scored high in self esteem were more likely to rate their general and mental health better (Table 2). HRQoL is subjective perception of one's health in various condition and thus sub-domains were expected to have positive association among them (9) as well as measure like self esteem.

Table 3 reports t-test results along with effect size of all sub-scales and self esteem scores between SUs and CGs. SUs scored poorer in all domains of SF-36 than the CGs. The magnitude of the effect size was largest for the RP sub-scale (effect size $=-0.79$ ) followed by GMH (effect size $=-0.76$ ) and SF (effect size $=-0.73$ ). The difference of BP scale however did not reach statistical significance. Regarding summary scales, widest gap was found for MCS (effect size $=-0.897$ ). 
Table 1. Socio-demographic characteristics of the sample.

\begin{tabular}{llll}
\hline Characteristics & Substance use \\
$(\mathrm{n}=120)$ & $\begin{array}{c}\text { Control group } \\
(\mathrm{n}=120)\end{array}$ \\
\hline Age (Mean, SD) & Male & $27.82(7.46)$ & $24.93(3.32)$ \\
Sex: $(\mathrm{n}, \%)$ & $95(79.2)$ & $58(48.3)$ \\
Education: $(\mathrm{n}, \%)$ & Female & $25(20.8)$ & $62(51.7)$ \\
& Up to SSC & $41(34.2)$ & $1(0.8)$ \\
& HSC & $13(10.8)$ & $18(15.0)$ \\
Family type: $(\mathrm{n}, \%)$ & Undergraduate & $41(34.2)$ & $43(35.8)$ \\
& Postgraduate & $25(20.8)$ & $58(48.3)$ \\
Socioeconomic status: & Joint & $80(66.7)$ & $79(65.8)$ \\
(n, \%) & Lower & $40(33.3)$ & $41(34.2)$ \\
& Middle & $9(7.5)$ & $73(60.8)$ \\
Birth order: $(\mathrm{n}, \%)$ & Upper & $94(78.3)$ & $44(36.7)$ \\
& Youngest & $17(14.2)$ & $3(2.5)$ \\
& Middle & $38(31.7)$ & $28(23.3)$ \\
& Eldest & $33(27.5)$ & $54(45.0)$ \\
\hline
\end{tabular}

Table 2. Mean SD and inter-relation among SF-36 sub-scales, and self-esteem.

\begin{tabular}{|c|c|c|c|c|c|c|c|c|c|c|c|c|}
\hline Variables & Mean (SD) & 1 & 2 & 3 & 4 & 5 & 6 & 7 & 8 & 9 & 10 & 11 \\
\hline $\mathrm{PF}$ & $\begin{array}{c}71.92 \\
(21.53)\end{array}$ & 1 & $0.337^{* *}$ & $0.476^{* *}$ & $0.395^{* *}$ & $0.534^{* *}$ & $0.250^{* *}$ & $0.497^{* *}$ & $0.344^{* *}$ & $0.732^{* *}$ & $0.499^{* *}$ & $0.162^{*}$ \\
\hline $\mathrm{RP}$ & $\begin{array}{c}53.37 \\
(30.04)\end{array}$ & & 1 & $0.338^{* *}$ & $0.436^{* *}$ & $0.429^{* *}$ & $0.461^{* *}$ & $0.406^{* *}$ & $0.324^{* *}$ & $0.766^{* *}$ & $0.564^{* *}$ & $0.336^{* *}$ \\
\hline BP & $\begin{array}{c}61.09 \\
(21.76)\end{array}$ & & & 1 & $0.514^{* *}$ & $0.510^{* *}$ & $0.308^{* *}$ & $0.442^{* *}$ & $0.244^{* *}$ & $0.704^{* *}$ & $0.542^{* *}$ & $0.153^{*}$ \\
\hline SF & $\begin{array}{c}58.85 \\
(22.52)\end{array}$ & & & & 1 & $0.511^{* *}$ & $0.508^{* *}$ & $0.476^{* *}$ & $0.374^{* *}$ & $0.603^{* *}$ & $0.788^{* *}$ & $0.248^{* *}$ \\
\hline GMH & $\begin{array}{c}59.55 \\
(19.78)\end{array}$ & & & & & 1 & $0.351^{* *}$ & $0.726^{* *}$ & $0.520^{* *}$ & $0.687^{* *}$ & $0.759^{* *}$ & $0.405^{* *}$ \\
\hline RE & $\begin{array}{c}57.22 \\
(38.01)\end{array}$ & & & & & & 1 & $0.316^{* *}$ & $0.306^{* *}$ & $0.480^{* *}$ & $0.804^{* *}$ & $0.297^{* *}$ \\
\hline VEF & $\begin{array}{c}54.49 \\
(20.10)\end{array}$ & & & & & & & 1 & $0.520^{* *}$ & $0.643^{* *}$ & $0.733^{* *}$ & $0.328^{* *}$ \\
\hline GHP & $\begin{array}{c}57.48 \\
(19.95)\end{array}$ & & & & & & & & 1 & $0.635^{* *}$ & $0.523^{* *}$ & $0.530^{* *}$ \\
\hline PCS & $\begin{array}{c}60.97 \\
(16.69)\end{array}$ & & & & & & & & & 1 & $0.747^{* *}$ & $0.412^{* *}$ \\
\hline MCS & $\begin{array}{c}57.53 \\
(19.51)\end{array}$ & & & & & & & & & & 1 & $0.403^{* *}$ \\
\hline $\begin{array}{l}\text { Self } \\
\text { esteem }\end{array}$ & $28.58(4.84)$ & & & & & & & & & & & 1 \\
\hline
\end{tabular}


The Table 4 shows comparison of HRQoL and self esteem of male and female participants of both groups separately. Males reported better in all sub-scales except RP and RE. For both groups, statistically significance difference was observed in case of PF, $\mathrm{BP}, \mathrm{GMH}$ and VEF sub-scales. Interestingly, the magnitude of the sex difference was higher for CGs compared to SU group. Significance sex difference of PCS and MCS scores were found only for the CG participants indicating females of CGs reported more deteriorated HRQoL than their male counterparts did. In case of SUs, sex difference was not profound.

Table 3. Difference of HRQoL and self esteem between substance use and control group.

\begin{tabular}{|c|c|c|c|c|c|c|c|}
\hline \multirow{2}{*}{ Variables } & \multicolumn{2}{|c|}{$\mathrm{SU}(\mathrm{n}=120)$} & \multicolumn{2}{|c|}{$C G(n=120)$} & \multirow{2}{*}{$\begin{array}{c}\text { Mean } \\
\text { difference }\end{array}$} & \multirow{2}{*}{$\mathrm{t}$} & \multirow{2}{*}{$\begin{array}{c}\text { Effect } \\
\text { size }^{* * *}\end{array}$} \\
\hline & Mean & $S D$ & Mean & $S D$ & & & \\
\hline PF & 68.34 & 24.150 & 75.50 & 17.933 & -7.16 & $-2.608^{*}$ & -0.39926 \\
\hline $\mathrm{RP}$ & 43.19 & 30.537 & 63.54 & 25.907 & -20.35 & $-5.566^{* *}$ & -0.7855 \\
\hline BP & 59.29 & 24.525 & 62.90 & 18.522 & -3.61 & -1.285 & -0.1949 \\
\hline SF & 51.56 & 22.662 & 66.15 & 19.939 & -14.59 & $-5.292^{* *}$ & -0.7317 \\
\hline $\mathrm{GMH}$ & 53.45 & 21.302 & 65.65 & 16.035 & -12.2 & $-5.0123^{* *}$ & -0.7608 \\
\hline RE & 45.56 & 37.157 & 68.89 & 35.301 & -23.33 & $-4.9874^{* *}$ & -0.66089 \\
\hline VEF & 51.18 & 21.982 & 57.81 & 17.485 & -6.63 & $-2.584^{*}$ & -0.37918 \\
\hline GHP & 54.21 & 17.947 & 60.75 & 21.342 & -6.54 & $-2.568^{* *}$ & -0.30644 \\
\hline PCS & 56.26 & 18.080 & 65.67 & 13.705 & -9.41 & $-4.545^{* *}$ & -0.68661 \\
\hline MCS & 50.44 & 20.330 & 64.62 & 15.800 & -14.18 & $-6.035^{* *}$ & -0.89747 \\
\hline Self esteem & 27.10 & 4.306 & 30.05 & 4.919 & -2.95 & $-4.943^{* *}$ & -0.59972 \\
\hline
\end{tabular}

${ }^{*} \mathrm{p}<0.05 .{ }^{* *} \mathrm{p}<0.001$. ${ }^{* * *}$ Effect size was calculated from the difference between group means and divided by control group $\mathrm{SD}^{(23)}$. A negative figure indicates $\mathrm{SU}$ participants mean worse than the control group mean.

To explore impact of addiction status (AS: SU versus CG) and self esteem on PCS and MCS, two way ANOVA were conducted. Participants were categorized into three equal percentiles groups based on their self esteem score (low: $<=26$, moderate: $27-30$ and high: 31 and above). In case of PCS, two way ANOVA revealed that there was significant main effect of AS, $F(1,234)=7.25, p<0.01, \eta^{2}=0.03$, indicating that overall CGs were better in PCS than SUs were. There was also significant main effect of self esteem $F(2$, $234)=12.15, p<0.001, \eta^{2}=0.094$. However, there was also significant interaction effect between AS and self esteem groups, $F(2,234)=3.38, \mathrm{p}<0.05, \eta^{2}=0.028$, which indicated that although SUs were lowered in PCS, the effect was different for different self esteem groups. Post-hoc comparisons indicated that the mean score of PCS for higher self esteem group $(M=69.72, S D=14.60)$ was significantly higher than moderate $(M=59.49$, $S D=14.27)$ and low self esteem group $(M=54.33, S D=17.38)$.

In case of MCS, two way ANOVA revealed almost similar results. There was significant main effect of AS, $F(1,234)=17.16, p<0.0001, \eta^{2}=0.068$. The effect size of the variance of MCS scores between two groups was moderate $\left(\eta^{2}=>0.06\right)$, but for PCS it 
was small $\left(\eta^{2}=>0.01\right)$. There was also significant main effect of self esteem $F(2,234)$ $=11.07, \mathrm{p}<0.0001, \eta^{2}=0.086$. The interaction effect did not reach statistical significance, $F$ $(2,234)=2.7, p>0.05, \eta^{2}=0.023$. Post-hoc comparisons indicated that the mean score of MCS for high self esteem group $(M=67.18, S D=15.58)$ was significantly higher than moderate $(M=56.32, S D=17.90)$ and low self esteem group $(M=49.80, S D=20.70)$. Similar analyses revealed no impact of SES, level of education, family type, occupation and birth order on either PCS or MCS.

Table 4. HRQoL and self-esteem of male and female participants.

\begin{tabular}{|c|c|c|c|c|c|c|c|}
\hline \multirow[t]{2}{*}{ Variables } & \multirow[t]{2}{*}{ Sex } & \multicolumn{3}{|c|}{$\begin{array}{c}\text { Substance user } \\
(\text { male }=95, \text { female }=25)\end{array}$} & \multicolumn{3}{|c|}{$\begin{array}{c}\text { Control group } \\
(\text { male }=58, \text { female }=62)\end{array}$} \\
\hline & & Mean & SD & $\mathrm{t}$ & Mean & SD & $\mathrm{t}$ \\
\hline \multirow{2}{*}{ PF } & Male & 70.69 & 23.882 & \multirow{2}{*}{$2.11^{*}$} & 80.86 & 17.043 & \multirow{2}{*}{$3.297^{* *}$} \\
\hline & Female & 59.40 & 23.511 & & 70.48 & 17.408 & \\
\hline \multirow{2}{*}{$\mathrm{RP}$} & Male & 42.37 & 30.526 & \multirow{2}{*}{-0.567} & 66.38 & 29.076 & \multirow{2}{*}{1.152} \\
\hline & Female & 46.33 & 31.002 & & 60.89 & 22.462 & \\
\hline \multirow{2}{*}{$\mathrm{BP}$} & Male & 61.61 & 23.395 & \multirow{2}{*}{$2.041^{*}$} & 69.05 & 18.637 & \multirow{2}{*}{$3.705^{* *}$} \\
\hline & Female & 50.50 & 27.147 & & 57.14 & 16.582 & \\
\hline \multirow{2}{*}{ SF } & Male & 51.97 & 21.751 & \multirow{2}{*}{0.386} & 68.53 & 19.342 & \multirow{2}{*}{1.273} \\
\hline & Female & 50.00 & 26.270 & & 63.91 & 20.384 & \\
\hline \multirow{2}{*}{ GMH } & Male & 55.62 & 20.115 & \multirow{2}{*}{$2.212^{*}$} & 71.90 & 13.670 & \multirow{2}{*}{$4.440^{* *}$} \\
\hline & Female & 45.20 & 23.993 & & 59.81 & 15.974 & \\
\hline \multirow{2}{*}{ RE } & Male & 45.96 & 36.443 & \multirow{2}{*}{0.234} & 67.82 & 37.962 & \multirow{2}{*}{-0.319} \\
\hline & Female & 44.00 & 40.506 & & 69.89 & 32.899 & \\
\hline \multirow{2}{*}{ VEF } & Male & 53.49 & 20.657 & \multirow{2}{*}{$2.284^{*}$} & 63.02 & 16.700 & \multirow{2}{*}{$3.285^{* *}$} \\
\hline & Female & 42.40 & 24.963 & & 52.93 & 16.907 & \\
\hline \multirow{2}{*}{ GHP } & Male & 54.48 & 18.220 & \multirow{2}{*}{0.316} & 64.20 & 21.750 & \multirow{2}{*}{1.729} \\
\hline & Female & 53.20 & 17.193 & & 57.52 & 20.605 & \\
\hline \multirow{2}{*}{ PCS } & Male & 57.29 & 17.927 & \multirow{2}{*}{1.215} & 70.12 & 14.595 & \multirow{2}{*}{$3.583^{* *}$} \\
\hline & Female & 52.36 & 18.496 & & 61.51 & 11.442 & \\
\hline \multirow{2}{*}{ MCS } & Male & 51.76 & 19.569 & \multirow{2}{*}{1.398} & 67.82 & 16.468 & $2175^{*}$ \\
\hline & Female & 45.40 & 22.718 & & 61.64 & 14.657 & $2.1 / 5$ \\
\hline Self esteem & Male & 26.87 & 4.074 & -1.123 & 29.86 & 5.017 & -0.403 \\
\hline & Female & 27.96 & 5.095 & & 30.23 & 4.860 & \\
\hline
\end{tabular}

${ }^{*} \mathrm{p}<0.05 .{ }^{* *} \mathrm{p}<0.01$.

SUs reported poorer score in all eight domains of HRQoL than the CGs. The magnitude of the effect size was largest for the RP followed by GMH and SF. SUs reported poorer mental well-being than CGs. These results are slightly different than the findings of $\mathrm{O}^{\prime}$ Brien et al.(5) and Millson et al.(10) .The magnitude of the difference was larger in existing studies than the current study. These variations might be attributed to the nature of the SUs, comparison norm and cultural context. SU participants of this 
study were generic in terms of drugs they addicted. Secondly, previous studies used normative data to compare SUs HRQoL. Future studies should consider larger normative sample while comparing HRQoL of SUs.

This study also revealed that males were better in physical areas while females reported less role limitation due to physical as well as emotional problems. In case of SUs, sex difference was not profound. This might be due to unequal sex distribution (male $79.2 \%$ ). In addition, it could be that the effect of substance might have equally affected both sexes with such strength that it diminished the existing difference of sex. Future research with equal number of sex might indicate different result.

This study reported variation in self esteem moderated HRQoL of both group with slightly higher magnitude for SUs. Both PCS and MCS were higher for those who scored high in self esteem than those who scored low and moderate. This finding is consistent with earlier studies ${ }^{(12,24,25)}$. Low self esteem individuals are usually affected with negative psychological aspects i.e., stress, depression. These in combination with harmful effect of substance might reduce HRQoL. This study did not find any effect of educational level, SES, family pattern, and birth order on HRQoL. This is significant in a sense that irrespective of socio-demographic characteristics impact of SU is equal to all. Service therefore should not vary based on any of the social phenomenon.

The findings of this study should be considered with some limitations. Firstly, participants were non-randomly taken, which might not be representative of this population. Secondly, present study did not take into consideration of types and duration of SU. Both of these might have differential impact on HRQoL. Future studies should target specific SU group with taking into consideration their period of use. Clinicians and psychologists should prioritize most affected areas such physical health, difficulties in social functioning and general mental health caused by SU when addressing well-being of this group. Initiatives to foster self esteem by incorporating psychological interventions, e.g., counselling, with the ongoing treatment might be useful. Further investigation is needed to know the changes of HRQoL over the period of treatment.

\section{References}

1. Murray CJL, T Vos, R Lozano, M Naghavi, AD Flaxman, C Michaud, M Ezzati, K Shibuya, AJ Salomon, S Abdalla, V Aboyans, J Abraham, I Ackerman, R Aggarwal and SY Ahn 2012. Disability-adjusted life years (DALYs) for 291 diseases and injuries in 21 regions, 19902010: A systematic analysis for the Global Burden of Disease Study 2010. The Lancet 380: 2197-2223.

2. Whiteford AH, L Degenhardt, J Rehm, AJ Baxter, AJ Ferrari, HE Erskine, FJ Charlson, RE Norman, AD Flaxman, N Johns, R Burstein, CJL Murray and T Vos 2013. Global burden of disease attributable to illicit drug use and dependence: Findings from the Global Burden of Disease Study 2010. The Lancet 382(9904): 1575-1586. 
3. Mn S, A Sj, M Msm, S Jua, A Smm and S Ahmed 2013. Drug addiction in Bangladesh and its effect. Medicine Today 25(02): 84-89.

4. González-Saiz F, O Rojas and I Castillo 2009. Measuring the impact of psychoactive substance on health-related quality of life: an update. Curr. Drug Abuse Rev. 4: 5-10.

5. O'Brien S, RP Mattick, J White, C Breen, J Kimber, A Ritter and N Lintzeris 2006. Maintenance pharmacotherapy for opioid dependence and SF-36 health status: A comparison with general population norms and other chronic disorders. Addictive Disorders \& Their Treatment 5(4):155-164.

6. Smith KW and MJ Larson 2003. Quality of life assessments by adult substance abusers receiving publicly funded treatment in Massachusetts. The American Journal of Drug and Alcohol Abuse 29(2):323-335.

7. Karow A, J Reimer, I Schäfer, M Krausz, C Haasen and U Verthein 2010. Quality of life under maintenance treatment with heroin versus methadone in patients with opioid dependence. Drug and Alcohol Dependence 112(3): 209-215.

8. Bullinger M 2003. Measuring health related quality of life, in Zouboulis C (ed). AdamantiadesBehçet's Disease. Advances in Experimental Medicine and Biology. Springer US.

9. Ware JE and CD Sherbourne 1992. The MOS 36-item short-form health survey (SF-36). I. Conceptual framework and item selection. Medical Care 30(6): 473-483.

10. Millson PE, L Challacombe, PJ Villeneuve, B Fischer, CJ Strike, T Myers, R Shore, S Hopkins, S Raftis and M Pearson 2004. Self-perceived health among canadian opiate users: A comparison to the general population and to other chronic disease populations. Canadian Journal of Public Health 95: 99-103.

11. Mann M, CMH Hosman, HP Schaalma and NK De Vries 2004. Self-esteem in a broad-spectrum approach for mental health promotion. Health Education Research 19(4):357-372.

12. Uba I, SN Yaacob, MA Talib, S Mofrad and R Abdullah 2013. Effect of self-esteem in the relationship between stress and substance abuse among adolescents: A mediation outcome. International Journal of Social Science and Humanity 3(3): 214-217.

13. Christo G and S Sutton 1994. Anxiety and self-esteem as a function of abstinence time among recovering addicts attending Narcotics Anonymous. British Journal of Clinical Psychology 33(2):198-200.

14. Akhter J 2012. Prevalence of substance abuse among female residential students of Dhaka University. ASA University Review 6(1).

15. Hasan M and ASM Shahin 2013. Drug Rehabilitation center based survey on drug dependence in Dhaka city. Update Dental College Journal 3(1): 32-36.

16. Riya S, M Rahman, MZ Sadeque, A Kabir and BU Umar 2013. Pattern of drug abuse among patients in some selected addiction rehabilitation centers in Dhaka city. Faridpur Medical College Journal 8(2): 63-66.

17. Feroz AHM, MN Islam, PM Ten Klooster, M Hasan, JJ Rasker and SA Haq 2012. The Bengali Short Form-36 was acceptable, reliable, and valid in patients with rheumatoid arthritis. Journal of Clinical Epidemiology 65(11): 1227-1235.

18. Ahmed SM, AKM Masud Rana, M Chowdhury and A Bhuiya 2002. Measuring perceived health outcomes in non-western culture: Does SF-36 have a place? Journal of Health Population and Nutrition 20(4): 334-342. 
19. Kurtin PS, AR Davies, KB Meyer, JM DeGiacomo and ME Kantz 1992. Patient-based health status measures in outpatient dialysis. Early experiences in developing an outcomes assessment program. Medical Care 30: 136-149.

20. Huque P and A Zaman 2002. Parental attitudes and adolescent's self esteem. Dhaka Univeristy Journal of Psychology 26: 19-26.

21. Rosenberg M 1989. Society and the adolescent self-image. Wesleyan University Press.

22. Blascovich J and J Tomaka 1991. Measures of Self-Esteem. In: Measures of Personality and social psychological attitudes. Measures of social psychological attitudes. Robinson JP, PR Shaver, LS Wrightsman and FM Andrews (eds). Academic Press.

23. Cohen J 1988. Statistical Power Analysis for the Behavioral Sciences. L. Erlbaum Associates.

24. Carvajal SC, SD Clair, SG Nash and RI Evans 1998. Relating optimism, hope, and self-esteem to social influences in deterring substance use in adolescents. Journal of Social and Clinical Psychology 17(4): 443-465.

25. Zamboanga BL, SJ Schwartz, LH Jarvis and K Van Tyne 2009. Acculturation and substance use among hispanic early adolescents: Investigating the mediating roles of acculturative stress and self-esteem. Journal of Primary Prevention 30: 315-333. 